\title{
BOOKS
}

\section{Handbook of infectious diseases}

Infections of the Central Nervous System, edited by Harold P Lambert (1991). BC Decker Inc, 320 Walnut Street, Suite 400, Philadelphia, Pennsylvania 19106, USA. ISBN 1-55664-206-7, 402 pages.

This is the first volume in an ambitious project initially promoted and developed by Ed Kass. His vision was for a multivolume series to encompass all infectious agents and describe major clinical complexes. The initial series was to deal with infection processes affecting organ systems, with plans for periodic revisions in the form of updated or new additions. This first volume, which discusses central nervous system infections, is edited by Harold P Lambert with many contributors, almost all of whom are British or American.

In 28 chapters the contributors cover in extraordinary detail aspects of infections that may affect the central nervous system. The goals of breadth and depth have generally been met, and the editors and contributors should be commended. This is a very useful and complete text, with most chapters exhaustively referenced. Of particular value are the full descriptions of some uncommon infections, including slow viruses, amoebic meningoencephalitis and protozoan and helminthic infections, as well as uncommon bacterial meningitides. In most areas this text provides more complete coverage than that in the current encyclopedic infectious diseases textbooks. An entertaining aspect is the historical perspective introducing each chapter which provides background and insight into these infections. Many chapters are entertaining reading, in particular, the chapter 'Meningococcal meningitis: Treatment' by Harold P Lambert and Robert A Wall.
Some aspects of the text can be criticized. The most problematic is that references beyond 1988 are not included. This reflects the prolonged incubation period for the 'Handbook of Infectious Diseases' series, with a great delay between completion of manuscripts and publication. Some deficits from this delay are the limited information with respect to conjugate vaccines for Hemophilus influenzae and clinical studies of the new azole antifungals in the treatment of meningitis. It is remarkable, however, how current and complete the volume remains despite this almost five-year lapse in citations. A second issue is why $H$ influenzae and Neissera meningitidis each warrant three chapters, while other infectious agents are dealt with in one chapter or less. The multichapter approach for these two agents, of necessity, leads to repetition among chapters and it is not clear what benefits it provides. Finally, the exceptionally complete and valuable chapter 'Penetration of antimicrobial agents into the central nervous system' by James W Stone and Richard Wise could benefit by the use of summary tables, and 'Neonatal meningitis' is not as completely referenced as other chapters. These, however, are minor problems in what is otherwise a useful and complete text.

This book is recommended for all infectious disease clinicians. It provides a complete, accessible review of these important infections, and can be used for detailed reference or day to day patient management issues. It would likely also be useful for general internists, pediatricians and neurologists. It is hoped that other volumes of the 'Handbook of Infectious Diseases' series will meet the high standard set by this initial volume.

LE Nicolle MD FRCPC Winnipeg. Manitoba 


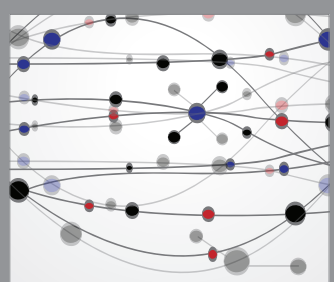

The Scientific World Journal
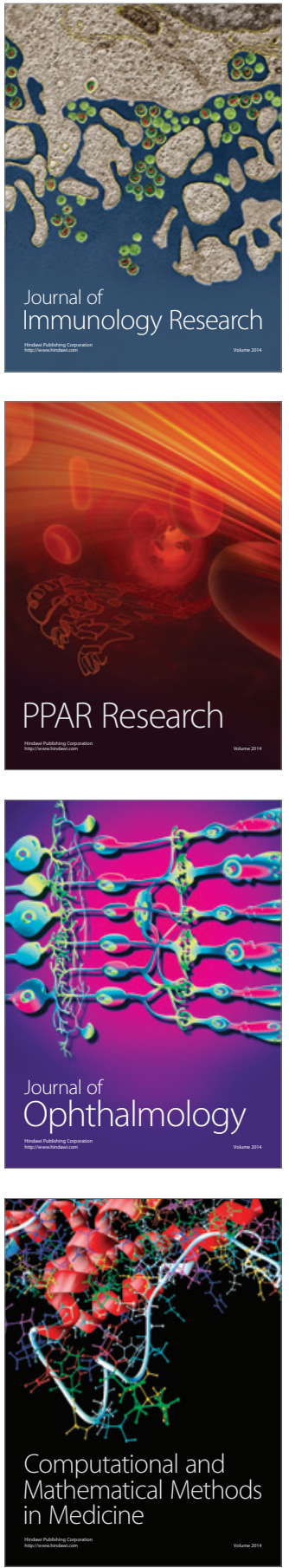

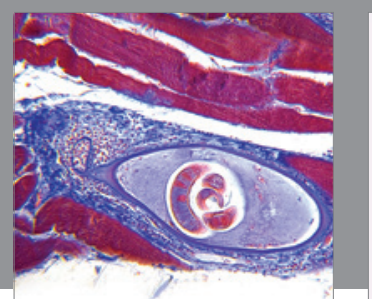

Gastroenterology Research and Practice

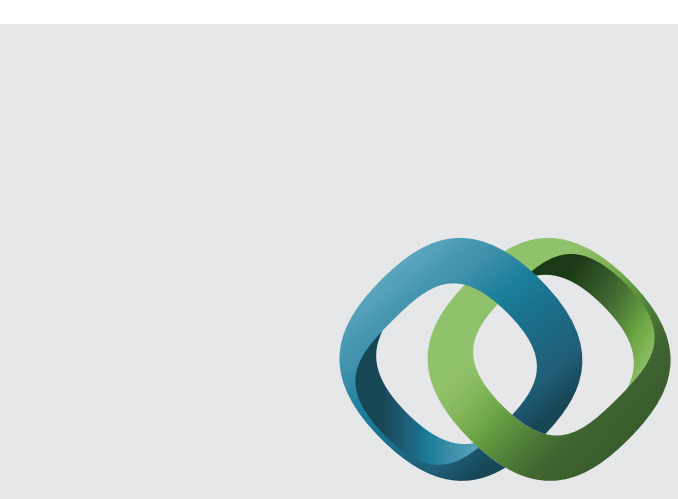

\section{Hindawi}

Submit your manuscripts at

http://www.hindawi.com
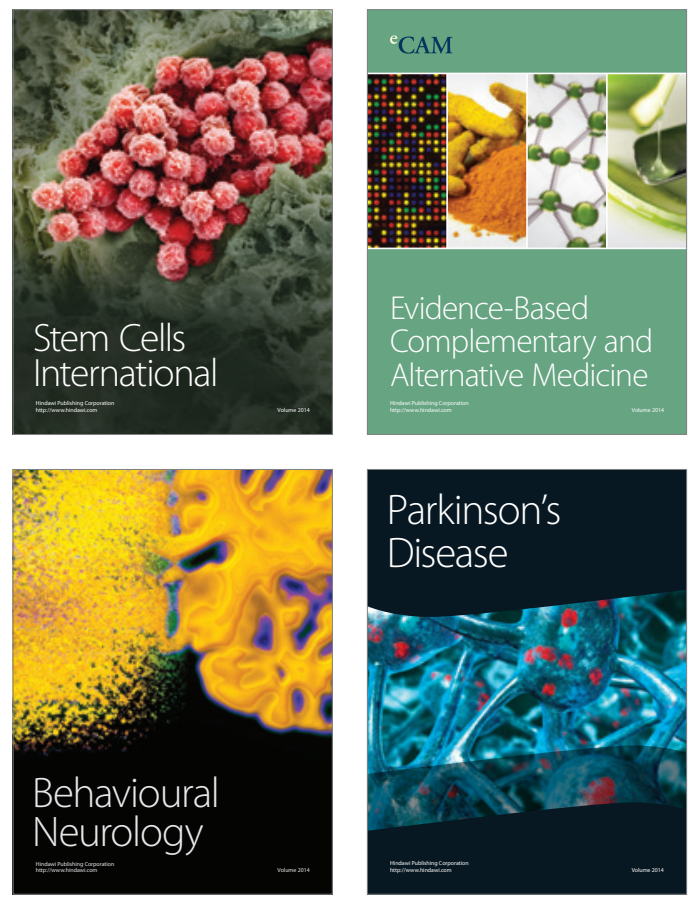
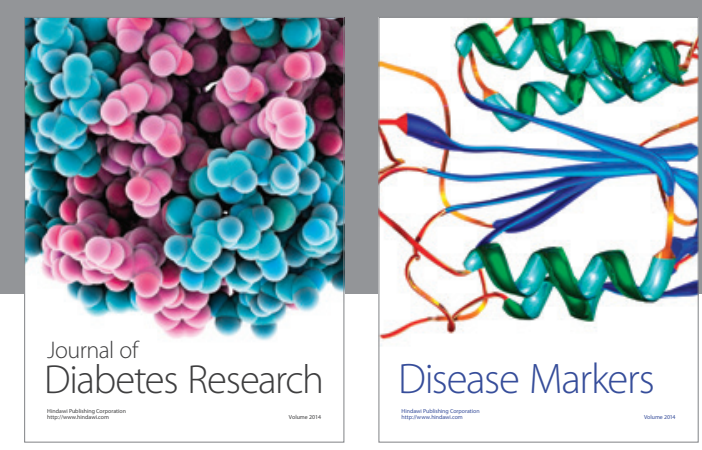

Disease Markers
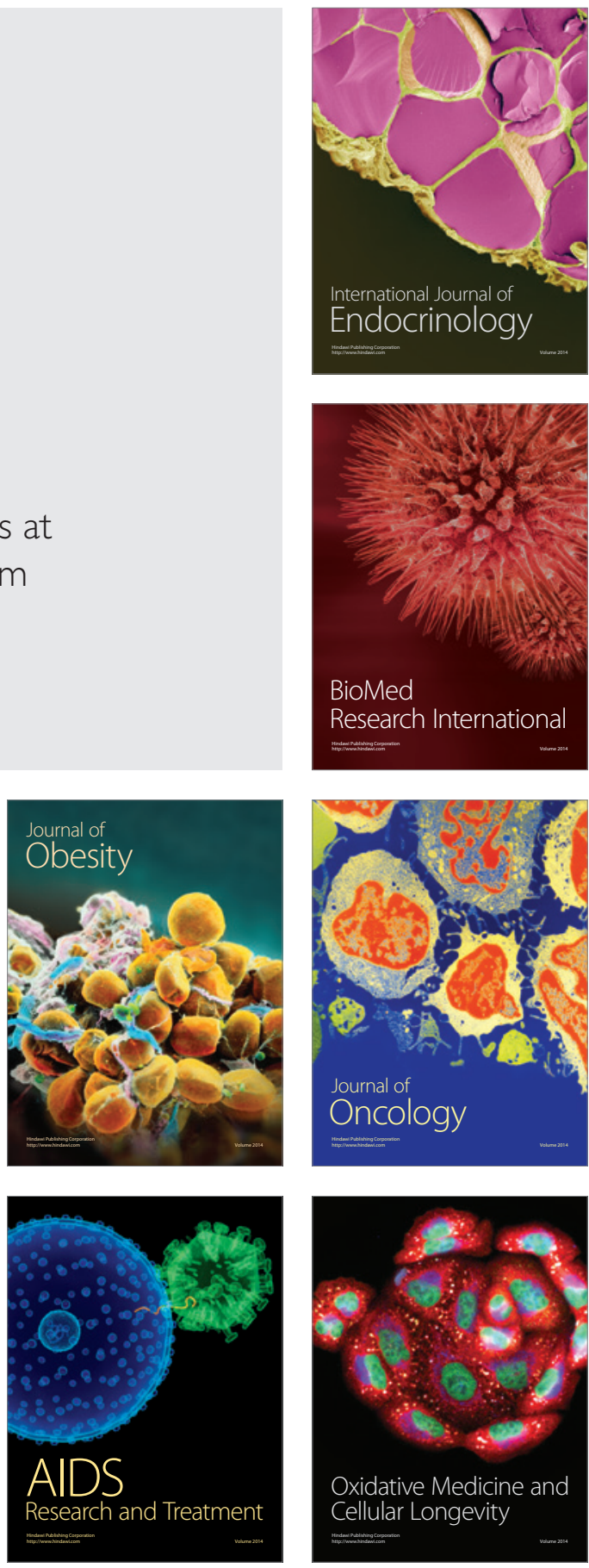